Effective electric fields along realistic DTI-based neural trajectories for modelling the stimulation mechanisms of TMS

This content has been downloaded from IOPscience. Please scroll down to see the full text. 2015 Phys. Med. Biol. 60453

(http://iopscience.iop.org/0031-9155/60/2/453)

View the table of contents for this issue, or go to the journal homepage for more

Download details:

This content was downloaded by: ndgeeter

IP Address: 31.5.75.17

This content was downloaded on 06/01/2015 at 15:21

Please note that terms and conditions apply. 


\title{
Effective electric fields along realistic DTI-based neural trajectories for modelling the stimulation mechanisms of TMS
}

\author{
N De Geeter ${ }^{1}$, G Crevecoeur $^{1,2}$, A Leemans $^{3}$ and L Dupré ${ }^{1}$ \\ 1 Department of Electrical Energy, Systems and Automation, Ghent University, \\ Technologiepark 913, 9052 Zwijnaarde, Belgium \\ 2 Department of Industrial Technology and Construction, Ghent University, \\ Campus Schoonmeersen, Valentin Vaerwyckweg 1, 9000 Ghent, Belgium \\ 3 PROVIDI Lab, Image Sciences Institute, University Medical Center Utrecht, \\ Heidelberglaan 100, 3584 CX Utrecht, The Netherlands
}

E-mail: Nele.DeGeeter@UGent.be

Received 16 July 2014, revised 9 November 2014

Accepted for publication 26 November 2014

Published 30 December 2014

\begin{abstract}
In transcranial magnetic stimulation (TMS), an applied alternating magnetic field induces an electric field in the brain that can interact with the neural system. It is generally assumed that this induced electric field is the crucial effect exciting a certain region of the brain. More specifically, it is the component of this field parallel to the neuron's local orientation, the so-called effective electric field, that can initiate neuronal stimulation. Deeper insights on the stimulation mechanisms can be acquired through extensive TMS modelling. Most models study simple representations of neurons with assumed geometries, whereas we embed realistic neural trajectories computed using tractography based on diffusion tensor images. This way of modelling ensures a more accurate spatial distribution of the effective electric field that is in addition patient and case specific. The case study of this paper focuses on the single pulse stimulation of the left primary motor cortex with a standard figure-of-eight coil. Including realistic neural geometry in the model demonstrates the strong and localized variations of the effective electric field between the tracts themselves and along them due to the interplay of factors such as the tract's position and orientation in relation to the TMS coil, the neural trajectory and its course along the white and grey matter interface. Furthermore, the influence of changes in the coil orientation is studied. Investigating the impact of tissue anisotropy confirms that its contribution is not negligible. Moreover, assuming isotropic tissues lead to errors of the same size as rotating or tilting the coil with 10 degrees. In contrast, the model proves to be less sensitive towards the not well-known tissue conductivity values.
\end{abstract}


Keywords: diffusion tensor imaging (DTI), effective electric field, primary motor cortex (M1), tractography, transcranial magnetic stimulation (TMS)

(Some figures may appear in colour only in the online journal)

\section{Introduction}

Transcranial magnetic stimulation (TMS) (Barker et al 1985) is a non-invasive technique that has the ability to enhance or suppress brain activity. With minimal discomfort, it induces electric currents and an electric field in the brain via a rapidly changing magnetic field, generated by a coil positioned above the scalp surface. This induced electric field interacts with the neural system and may excite or inhibit certain brain regions by altering the membrane potentials of neurons (Roth and Basser 1990). Over recent years, TMS has established itself as a promising diagnostic tool and alternative treatment for a broad range of neurological and psychiatric disorders (Ilmoniemi et al 1999) including strokes (Wagner et al 2006), Parkinson's disease (Fregni et al 2005), tinnitus (Smith et al 2007), epilepsy, phantom pain and depression (Padberg and George 2009).

Although during the last decade TMS has been frequently and widely used and its efficacy has already been shown, the underlying mechanisms remain poorly understood (Sack and Linden 2003) and its working is hard to predict. Moreover, the treatment works well for some patients, for others only temporary, and for others not at all (Loo and Mitchell 2005). This uncertainty restricts the many other potential applications and keeps neuroscientists from embracing TMS as a routine treatment method. To gain more insight, research is needed including bio-electromagnetic modelling that can accurately determine the impact of electromagnetic fields on the human brain both qualitatively as quantitatively. These simulations are crucial because in vivo measurements are typically expensive and large homogeneous patient groups are difficult to gather and restricted due to ethical issues (Rossi et al 2009).

Research in the field of TMS modelling has been conducted in various constructive phases. The magnetic field produced by the stimulation coil and the induced electric field were first calculated (Cohen et al 1990, Thielscher and Kammer 2004, Salinas et al 2007). The induced eddy currents were further considered by Sekino and Ueno (2004), Wagner et al (2004) and De Geeter et al (2011a) to quantify the impact of TMS. Finally, the stimulation parameters which are related to the electric field along neural fibres were preliminary studied by Lu et al (2008), Salvador et al (2011), Pashut et al (2011) and Opitz et al (2011) among others. We believe that these latter electromagnetic computations need to be scrutinized and more accurately explored to fully understand and optimize the use of TMS.

In this paper, we introduce a flexible and personalized TMS modelling method. First, the induced electric field distribution in the whole brain is calculated using a recently developed electromagnetic solver presented by De Geeter et al (2012). Secondly, this electric field is mapped along the trajectories of the main neural fibre bundles and serves as the input for the stimulation mechanisms. Personalized head models are used and include realistic geometry and frequency dependent anisotropic tissue properties, i.e. conductivity and permittivity, based on T1-weighted magnetic resonance images (MRI) and diffusion-tensor MRI (DTI). DTI exploits the directional dependent diffusion of water molecules in the brain, with the principal diffusion direction corresponding to the predominant orientation of fibre bundles (Jones and Leemans 2011). Including these features is important, since Janssen et al (2013), De Lucia et al (2007) and Miranda et al (2007) showed that even small geometrical alterations in the cortex as well as anisotropy and tissue heterogeneity can alter significantly the field distribution. The coil configuration can be chosen freely and is specified by the coil shape, 
stimulation pulse and position to the head and region of interest, which is in contrast to Pashut et al (2011) not limited to a plane parallel to that of the coil. Another restriction in the previously performed studies, is that a simplified geometry of the neural trajectories is considered and the respective electric field component along these bundles. In Lu et al (2008), the $x$-, $y$ - and $z$-components of the electric field are computed and investigated for a non realistic straight test line within the brain. An artificial fibre mimicking a two-dimensional (2D) axon reconstruction of the primary motor cortex based on macaque monkey data is considered in Opitz et al (2011). Salvador et al (2011), on the other hand, modelled several types of neurons within a human folded cortical sheet to study the activation mechanisms with a 2D geometry of the trajectories. In contrast, this paper employs tractography based on the diffusion tensor framework of the patient, to generate realistic 3D reconstructions of the neural fibres in vivo. Whole brain tractography is performed, using a deterministic streamline approach, to trace all neural fibre trajectories (Basser et al 2000, Leemans et al 2009). Close to the brain region of interest that is targeted, bundles of grouped neural fibres, called tracts, are selected. This flexible and personalized way of TMS modelling is, to our knowledge, new for the simulation of the response to TMS and a valuable step towards fully case- and patient-specific results.

Integrating DTI information in TMS is a relatively new concept. The diffusion tensor has mainly been used to derive realistic fibre anisotropy (De Lucia et al 2007, De Geeter et al 2012). In two recent studies of Opitz et al (2011) and Nummenmaa et al (2014) DTI-based tractography is applied to model the geometry of neural bundles. Opitz considered a small number (tens) of similarly oriented tracts close to each other, while Nummenmaa included about 750 detailed tracts. Both studies focussed on short (several $\mathrm{mm}$ ) neural tracts localised near only one gyrus, whereas we study a substantially larger region of interest. Furthermore, the latter computed the electric field distribution inside the skull, using a simplified singlecompartment boundary element model (BEM) without incorporating anisotropic conductivities. Moreover, they investigated the absolute value of the induced electric field gradient, i.e. the derivative of the electric field component parallel to the tract. This term is only one of the three stimulation mechanisms as described in Silva et al (2008); and by neglecting the sign, distinction between depolarization and hyperpolarization cannot be made. In Shahid et al (2014), both tissue anisotropy and fibre tract information were subtracted from DTI to model the effects of transcranial direct current stimulation (tDCS).

The case study of this paper focuses on the stimulation of the hand area of the left primary motor cortex (M1). We study the importance of implementing realistic 3D neural trajectories and the effect of small changes in orientation of the considered neural fibre bundles on the stimulation mechanisms. We quantitatively investigate the impact of the poorly-known values of the tissue conductivities and neglecting their anisotropic properties. Moreover, the sensitivity of stimulation towards small adaptations in the coil configuration is examined.

\section{Methodology}

In this section we explain the modelling of the electromagnetic effects of a TMS excitation pulse on a human head and the inclusion of the neural fibres through the use of accurate computer simulations.

\subsection{Realistic patient-specific head model}

An accurate personalized head model is created from structural and diffusion-weighted MRI. It includes realistic geometry, frequency dependent electrical properties, i.e. conductivity and 

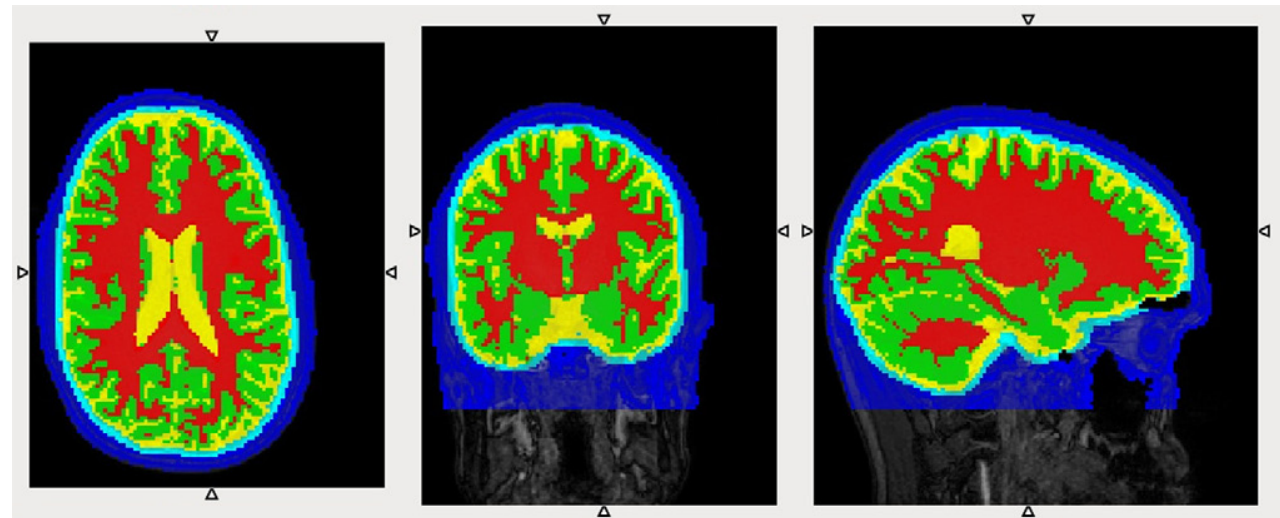

Figure 1. Segmentation based on T1-weighted MRI. Transverse (left), coronal (middle) and sagittal (right) view of the constructed head model containing the tissues scalp (blue), skull (turquoise blue), cerebrospinal fluid (yellow), grey matter (green) and white matter (red) and surrounded by air (black). The original T1-weighted image is visualized on the background.

permittivity, tissue anisotropy and traced neural fibre bundles connected with the targeted region.

A T1-weighted image was acquired from a healthy volunteer (female, $25 \mathrm{y}$ ) on a 3 Tesla MR scanner (Achieva 3.0 T, Philips) using a turbo field-echo sequence $(\mathrm{TR} / \mathrm{TE}=25 / 4.5 \mathrm{~ms}$, flip angle $=30^{\circ}$, field of view (FOV) $160 \times 240 \times 240 \mathrm{~mm}^{3}$ and matrix size $160 \times 256 \times 256$ ) The subject gave informed consent to participate in this study under a protocol approved by the University Medical Center Utrecht ethics board. Statistical Parametric Mapping (SPM8) (Friston et al 2007) was used to segment this image into tissue probability maps. Based on these maps, a head model is constructed (FOV $192 \times 236 \times 206 \mathrm{~mm}^{3}$ and matrix size $96 \times 126 \times 110$ ), surrounded with air and segmented into scalp, skull and the cerebral tissues cerebrospinal fluid, grey and white matter as visualized in figure 1 . The electrical properties of these tissues vary naturally with frequency and their isotropic values are obtained from the 4-Cole-Cole model (Cole and Cole 1941, Gabriel et al 1996).

White matter is known to be anisotropic due to the electrically insulating myelin sheaths. Since the transport of ions in conductivity and water molecules in diffusion are mainly constrained by the same extracellular space, a strong linear relationship between the eigenvalues of the conductivity and the self-diffusion tensors exists (Tuch et al 2001). Therefore, in addition to the T1-weighted image, a DTI data set of the same subject was acquired using a single-shot spin-echo EPI (Echo Planar Imaging) sequence (FOV $224 \times 224 \mathrm{~mm}^{2}$ and matrix size $128 \times 128$, 60 diffusion-weighted images with a b-value of $1200 \mathrm{~s} \mathrm{~mm}^{-2}$ and 1 non-diffusion-weighted image $(\mathrm{NEX}=6)$ ) (Jones and Leemans 2011). The diffusionweighted MRI data were corrected for (i) subject motion and eddy current induced geometric distortions (Leemans and Jones 2009) and (ii) EPI based deformations due to magnetic field inhomogeneities by non-rigidly warping the diffusion data to the T1-weighted image (Irfanoglu et al 2012). The diffusion tensor was estimated using a robust outlier detection procedure (REKINDLE) with the iteratively reweighted linear least squares approach (Veraart et al 2013, Tax et al 2014). Anisotropic conductivity and permittivity tensors were computed from this diffusion tensor (De Geeter et al 2012). It is important to incorporate these anatomical details, tissue heterogeneity and anisotropy, since they can significantly 



Figure 2. Selection of the five main neural fibre tracts from the group of 54 tracts near the left M1 hand area. Two extra fibres with similar oriented trajectories are selected, marked with an asterisk and indicated with dotted lines. The blue dot is the seed ROI centre.

alter the induced electric field distribution (De Lucia et al 2007, Miranda et al 2007). For more details on the constructed head model matrix and its reference space, we refer to the appendix A.

\subsection{Realistic oriented trajectories of neural fibre bundles}

Tractography (Basser et al 2000) is a relative new method based on DTI that can generate anatomically plausible and reproducible reconstructions of fibre tracts in the human brain in vivo. In this paper, the graphical toolbox ExploreDTI (Leemans et al 2009) is used. First, a whole brain tractography is performed, in which a deterministic tracking algorithm estimates the principal diffusion orientation at each voxel, functioning as seed points, and propagates along this direction in steps of $1 \mathrm{~mm}$. The seed fractional anisotropy (FA) measures the degree of preference for a single diffusion direction and its threshold is set to 0.2 . The bending angle threshold is set to 30 degrees. Subsequently the region of interest (ROI) method is applied to extract the relevant neural fibres. The left M1 hand area is defined as the central seed ROI, depicted as the blue dot in figure 2 , and within its $15 \mathrm{~mm}$ range a group of about 50 neural tracts between 5 and $25 \mathrm{~cm}$ is selected. From this group, the five most common and representative tracts are detected where we focus our study upon. Another two are selected with trajectories similarly oriented to those of tract 1 and 2, see figure 2. Data from ExploreDTI was exported and post processed in Matlab (R2013a, The Mathworks, Inc. Natick, MA, USA). More details about the selection method can be found in the appendix A.

All tracts are considered as myelinated fibre bundles connecting one grey matter-white matter (GM-WM) interface with another, as illustrated in figure 3 for tract 1 and 4 . These bundles thus mainly contain pyramidal neurons. When the axons of these neurons form part of the pyramidal tracts leading to the spinal cord or brain stem, they are so-called pyramidal tract neurons (PTN), whereas neurons with their axons primarily sent to other cortical areas are pyramidal association fibres. The non-pyramidal neurons are called interneurons and run through the cortical surface without crossing the GM-WM interface. Tract 1, 2, (1* and $\left.2^{*}\right)$ are PTNs whereas tract 3-5 are association fibres. We make this distinction to investigate if we can draw similar conclusions as Salvador et al (2011) concerning the activation mechanisms. 

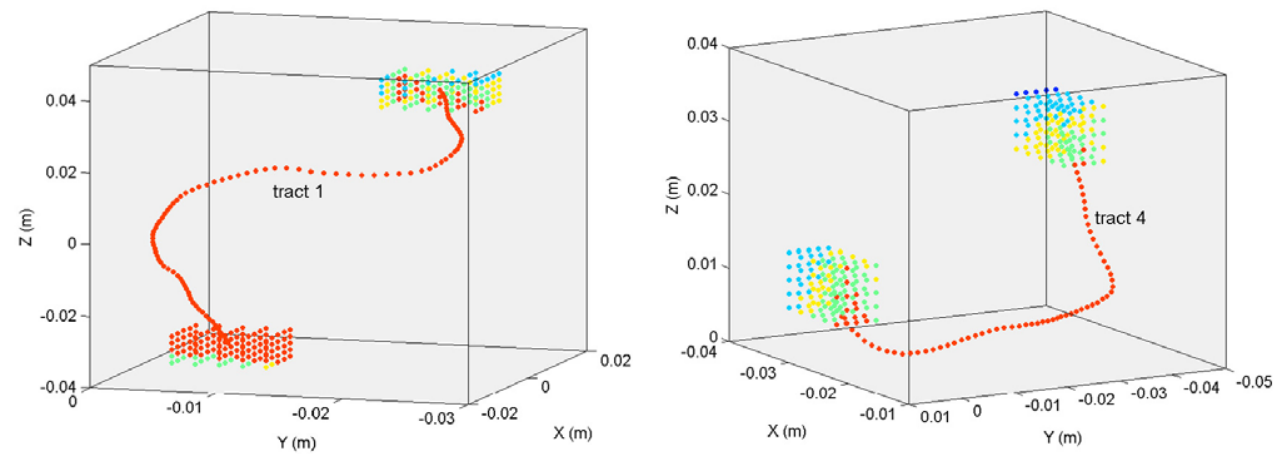

Figure 3. Fibre tracts connecting one GM-WM interface with another. (Left) Tract 1 and (right) tract 4 with the surrounding $5 \times 5 \times 5$ voxels near the first and last points of the discretized fibre. The colours represent the corresponding tissue labels, analogously as in figure 1 .
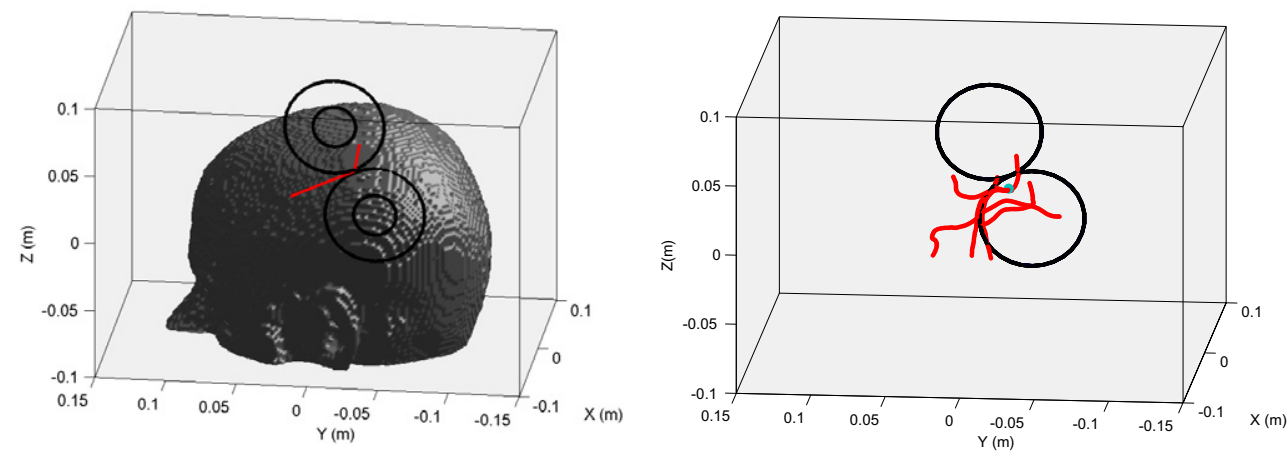

Figure 4. Coil location and orientation for TMS of the left M1 hand area. (Left) The coil position with respect to the head model. Both the inner and outer windings of the $70 \mathrm{~mm}$ figure-of-eight biphasic Nexstim coil are visualized. The red lines illustrate the coil normal and the induced current direction. (Right) The coil position, with respect to the selected neural fibre tracts and the seed ROI centre, respectively, represented by red lines and a blue dot. Only the outer windings of the coil are plotted for clarity reasons.

\subsection{TMS coil configuration}

The TMS configuration can be characterized by the following parameters: the coil position, orientation and its design, the pulse waveform, duration and frequency and the stimulus amplitude. The presented method is developed in a flexible way so that all these parameters can be varied freely. The simulations are not restricted to coils placed parallel to the plane of the neural fibre bundle, as was the case in Pashut et al (2011). Remark that the considered neural bundles, depicted in figures 2 and 3, are three dimensional and thus not situated in a plane.

We model the response of a single biphasic pulse with a sinusoidal waveform and a pulse width of $230 \mu$ s, delivered to the hand area of the left primary motor cortex. The $70 \mathrm{~mm}$ figureof-eight Nexstim coil (Nexstim Ltd., Helsinki, Finland) is applied, with a mean winding diameter of $50 \mathrm{~mm}$, and modelled as an air-core coil. During stimulation, it is placed tangentially to the scalp surface and the maximal induced current is directed anteriorly, as shown in figure 4 . 


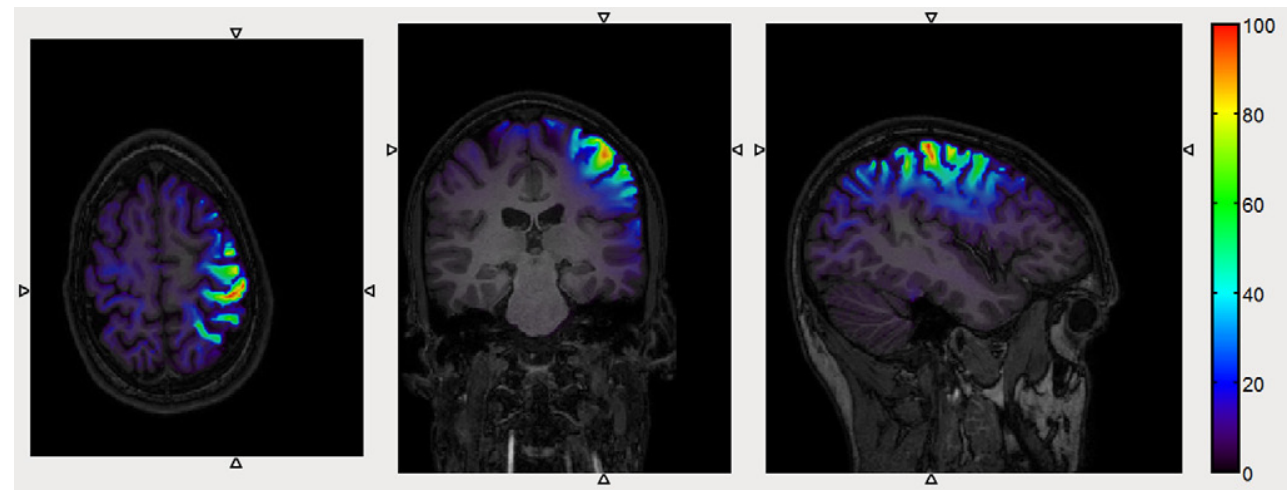

Figure 5. Induced electric field magnitude distribution (in $\mathrm{V} \mathrm{m}^{-1}$ ) due to TMS of the left M1 hand area. Only the values in the cerebral tissues are visualized for clarity reasons. Note that the views are different from those in figure 1.

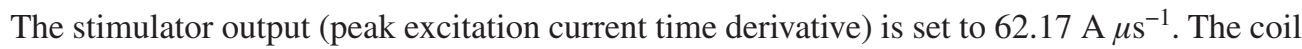
can be rotated and tilted to study the sensitivity of the stimulation mechanisms with respect to the coil's orientation.

\subsection{The electric field and corresponding stimulation mechanisms}

The recently developed anisotropic independent impedance method (IIM) is used for performing the electromagnetic simulations, described in De Geeter et al (2011a, 2011b). IIM is based on the conventional IM, but with the dependent loops and consequently redundant equations (Wang et al 2008) eliminated resulting in improved conditionality and speed up of numerical convergence. A 3D network of impedances is generated and solved to determine the induced eddy currents. The electric field $E$ distribution over the whole brain can then be calculated as in De Geeter et al (2012). Since we are mainly interested in the electric field distribution within the cerebral tissues, only those voxels are imaged in figure 5.

The component of this electric field tangent to the trajectory $l$ of each selected neural tract is then computed and depicted in figure 6. This term is called the effective electric field $E_{l}$, since it effectively contributes to neuronal stimulation, expressed in three different stimulation mechanisms (Silva et al 2008). The first mechanism is defined as the directional derivative of the effective electric field along the neural fibre and equals

$$
-\lambda^{2} \frac{\partial E_{l}}{\partial l}
$$

This term was initially assumed to describe the effect of stimulation by electromagnetic induction on the membrane potential $V$ relative to its resting value, better known as the cable equation (Roth and Basser 1990)

$$
\lambda^{2} \frac{\partial^{2} V}{\partial l^{2}}-V=\tau \frac{\partial V}{\partial t}+\lambda^{2} \frac{\partial E_{l}}{\partial l} .
$$

$\lambda$ is the length constant of the neural membrane, $\tau$ the time constant and $l$ the local space parameter along the neural fibre. Silva et al (2008) introduced a second mechanism, occurring at terminations and sharp bends of the fibre 


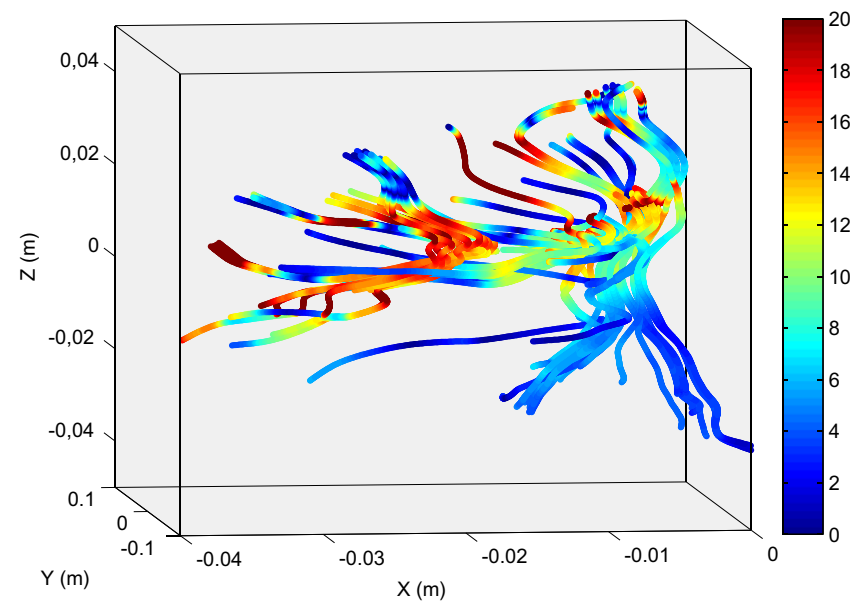

Figure 6. Induced effective electric field magnitude (in $\mathrm{V} \mathrm{m}^{-1}$ ) along all 54 neural fibre bundles due to TMS of the left M1 hand area. The colour limit is set to $20 \mathrm{~V} \mathrm{~m}^{-1}$ for clarity reasons.

$$
-\lambda E_{l}
$$

even in the absence of effective electric field gradients, and a third jump mechanism at the GM-WM interface due to the changing conductivity values (Miranda et al 2007)

$$
-\lambda \frac{\Delta E}{2}
$$

Since large tracts longer than $5 \mathrm{~cm}$ are considered in this paper, the length constant $\lambda$ is set to $2 \mathrm{~mm}$ (Silva et al 2008). Figure 7 schematically illustrates the three different stimulation mechanisms and the sites where they occur, for a certain neural fibre bundle. These three mechanisms (1), (3) and (4) were tested on synthetic neural fibres by Salvador et al (2011) and we will now simulate them on realistic human fibre tracts.

\section{Results}

\subsection{Effective electric field}

This paper models and simulates the effect of single pulse stimulation delivered to the left M1 hand area, with coil position according to figure 4, using patient-specific MR data (figures 1 and 2). For this case study, the induced electric field is computed in the whole brain (figure 5) and the effective electric field is studied along the DTI-based realistic neural fibre trajectories (figure 6), since this is the source term of the cable equation and the three stimulation mechanisms (1)-(4). All results are plotted at the time instant corresponding with maximum excitation current.

While the electric field in figure 5 remains mainly concentrated in the vicinity of the coil, its component parallel to the neuron's local orientation exhibits a larger and more irregular spatial spread, as can be seen from the $40 \times 20 \times 100 \mathrm{~mm}^{3}$ box in figure 6 . This clarifies our decision to consider a substantially larger area of interest than previous studies, namely all tracts traversing the $15 \mathrm{~mm}$ range around the left M1 hand hotspot. More details can be found in the appendix A. Not in all tract points near the coil, and thus near the M1 ROI, a 




Figure 7. Neural fibre bundle and corresponding stimulation mechanisms. The $l$-axis is lying along the trajectory of the neural fibre and the effective electric field $E_{l}$ is the component of the induced electric field $E$ tangent to this axis.



Figure 8. Strong and localized variation of the effective electric field. For each tract point, the effective field magnitude (in $\mathrm{V} \mathrm{m}^{-1}$ ) is plotted in function of its distance from the M1 ROI centre (in $\mathrm{m}$ ). The colours indicate the number of tract points out of a total of 41664 points with those parameters. The averaged effective field over $1 \mathrm{~mm}$ intervals is represented by the red line.

substantial effective electric field is induced, and at the same time it is not limited to those points. This is illustrated more quantitatively in figure 8 , in which the effective electric field of each point in the 54 neural tracts is plotted as a function of the distance between this point and the seed ROI centre. A strong and localized variation of the field can be observed. This is caused by the interplay of different factors such as the tract's position and orientation in relation to the TMS coil, the neural trajectory and its course along the white and grey matter interface. At a distance of $20 \mathrm{~mm}$, the effective electric field can be near to zero or $25 \mathrm{~V} \mathrm{~m}^{-1}$, illustrating the dependence on the above mentioned various factors. The same can be observed at $40 \mathrm{~mm}$. Moreover, the average effective field is not continuously descending for increased distance: $14 \mathrm{~mm}$ away from the $\mathrm{M} 1$ hotspot, the field amounts on average $9.6 \mathrm{~V} \mathrm{~m}^{-1}$, while at a double distance it is $12.9 \mathrm{~V} \mathrm{~m}^{-1}$. At single tract points, this effect is 

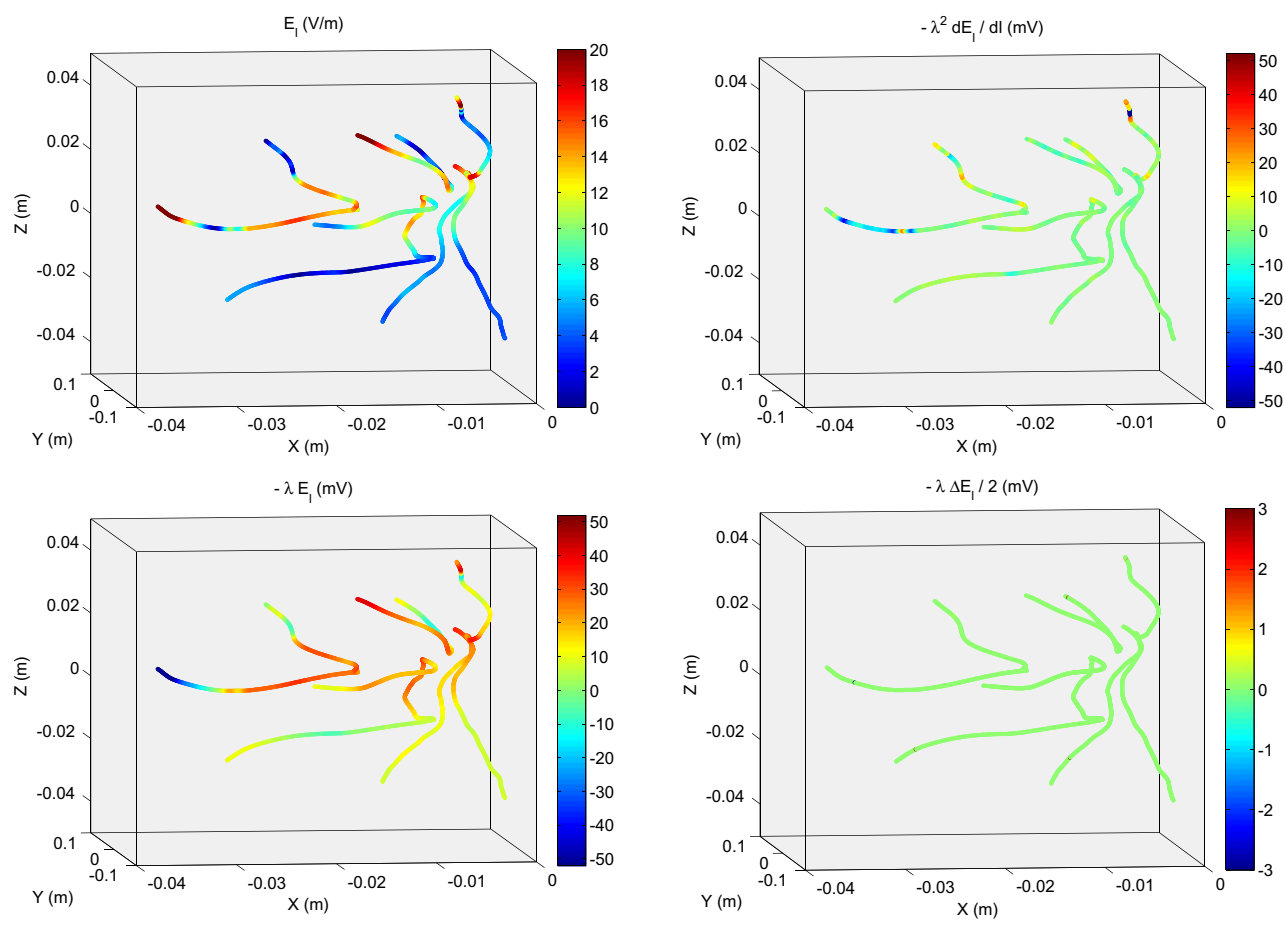

Figure 9. Modelling the impact of left M1 hand area TMS on the five considered neural fibre bundles. (Upper left) Induced effective electric field magnitude (in $\mathrm{V} \mathrm{m}^{-1}$ ). The colour limit is set to $20 \mathrm{~V} \mathrm{~m}^{-1}$, similar as in figure 6. (Upper right) Directional derivative of the effective electric field along the neural fibres (in $\mathrm{mV}$ ). (Lower left) Effective electric field term (in $\mathrm{mV}$ ) which mainly reaches higher values at terminations and sharp bends. (Lower right) Electric field jump mechanism at the GM-WM interface (in $\mathrm{mV})$. The colour range of the first two stimulation mechanisms is set to $[-52,52] \mathrm{mV}$, equally to the stimulation threshold, the third one to $[-3,3] \mathrm{mV}$ for clarity reasons. The length constant $\lambda$ is equal to $2 \mathrm{~mm}$.

even more pronounced, with effective field values up to $31.8 \mathrm{~V} \mathrm{~m}^{-1}$ at $27 \mathrm{~mm}$ distance and $17.1 \mathrm{~V} \mathrm{~m}^{-1}$ at $49 \mathrm{~mm}$. The occurrence of the effective field amplitude at certain distance gives a trend of its spatial extend.

The order of electric field magnitude decreased from $100 \mathrm{~V} \mathrm{~m}^{-1}$ (figure 5) to tens of $\mathrm{V} \mathrm{m}^{-1}$ for its effective component (figures 6-8), which is consistent to literature for the applied stimulator output of $62.17 \mathrm{~A} \mu \mathrm{s}^{-1}$ (Miranda et al 2007, Salvador et al 2011).

In figure 9 we focus on the effective electric field along the five considered neural tracts and the corresponding 3 stimulation mechanisms. These mechanisms interact with the voltage-gated ion channels at the plasma membrane of the neurons, in accordance with the Hodgkin-Huxley dynamics and alter the membrane potentials (Roth and Basser 1990). Once they exceed a threshold, depolarization occurs and an action potential is initiated resulting in an excited or inhibited neuron. Similar as in Silva et al (2008), we assume stimulation will be achieved when one of the mechanisms reaches an amplitude higher than $52 \mathrm{mV}$. Remark no mechanism for the five selected tracts exceeds $52 \mathrm{mV}$ and consequently no action potential takes place. Nevertheless, these figures show relative results with respect to the stimulator output of this case study. When increasing the intensity of the stimulator, and thus the excitation current amplitude, the induced electric field increases proportionally and the stimulation 

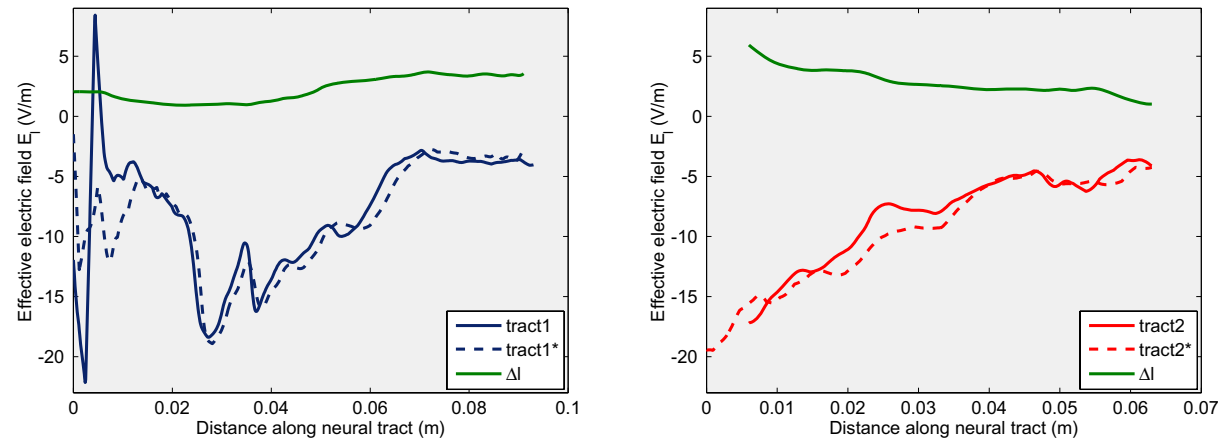

Figure 10. Effect of considering a slightly different oriented tract on the spatial variation of the effective electric field (in $\mathrm{V} \mathrm{m}^{-1}$ ). (Left) Tract 1 and (right) 2 with their corresponding similar tracts. $\Delta l$ is the distance (in $\mathrm{mm}$ ) between both neural tracts. The distance is measured starting from the point closest to the M1 ROI.

mechanisms will exceed the stimulation threshold. The effective electric field term $-\lambda E_{l}$ is the most dominant mechanism. It reaches maximal values of $44.31 \mathrm{mV}$ for tract $1,34.43 \mathrm{mV}$ for tract 2 and $41.89 \mathrm{mV}$ for tract 5, all within the first $2.5 \mathrm{~mm}$, and 33.04 and $33.10 \mathrm{mV}$ for tract 3 and 4 at the place where they bend sharply. So this mechanism would exceed the $52 \mathrm{mV}$

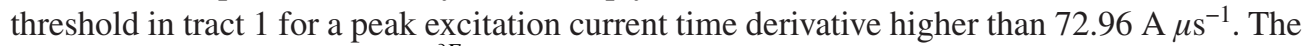
directional derivative term $-\lambda^{2} \frac{\partial E_{l}}{\partial l}$ operates primarily in tract 1 and 4 , with voltages up to 29.91 and $26.06 \mathrm{mV}$, due to the fast changing field at those points. The jump term $-\lambda \frac{\Delta E}{2}$ remains small $(<3 \mathrm{mV})$ for all tracts in this case study. In general, we can state that the stimulation mechanisms are the highest at neural terminations and bends; and that therefore activation mainly occurs at those sites.

These findings of strong variation and the preferred stimulations sites are consistent with those of Salvador et al (2011). However, we find it hard to determine which mechanism dominates for which type of tract (PTNs 1, 1*,2, 2*; association fibres 3-5) and even to dissociate these different types. This can be explained by the fact that in contrast to including predefined and simplified neural pathways, we received realistic, but irregular geometrical information from the DTI-based tractography.

\subsection{Differently oriented neural tracts}

In this subsection we assess the effect of differently oriented neural tracts on the effective electric field. As previously mentioned, alternative but closely located tracts were selected for tract 1 and 2 and marked with an asterisk ( $1 *$ and $2 *)$. Their effective electric fields are plotted in figure 10 as function of the distance along the neural pathway.

The distance $l$ along neural tract 1 is measured from coordinate $(-6.25,-24.52,41.80)$ in mm towards $(-1.00,-6.69,-34.50)$. The difference in effective electric field $\Delta E_{l}$ between this tract and its alternative has a mean of $0.52 \mathrm{~V} \mathrm{~m}^{-1}$ and reaches its maximum of $15.92 \mathrm{~V} \mathrm{~m}^{-1}$ for $l$ equal to $4.4 \mathrm{~mm}$. The point-wise difference $\Delta l$ between the coordinates of both amounts to a minimum of $0.9 \mathrm{~mm}$, a maximum of $3.7 \mathrm{~mm}$ and is on average $2.2 \mathrm{~mm}$. The largest $\Delta E_{l}$ occur mainly in the first $5 \mathrm{~mm}$ of the tract, whereas $\Delta l$ in this interval is limited to $2 \mathrm{~mm}$ and the angle between both tract orientations varies between 0.8 and 4 degrees. This difference in effective field even causes the dominant stimulation mechanism to change from $-\lambda E_{l}$ for tract 



Figure 11. Effect of neglecting tissue anisotropy on the spatial variation of the effective electric field (in $\mathrm{V} \mathrm{m}^{-1}$ ). (Upper left) The anisotropic and isotropic results are indicated with solid and dotted lines, respectively. (Upper right) The difference between both The distance is measured starting from the point closest to the M1 ROI. (Lower left) Box plots of the effective electric field for both cases (in $\mathrm{V} \mathrm{m}^{-1}$ ) for all 54 tracts. (Lower right) Box plot of the error $\Delta E_{\text {liso }}$ (in $\mathrm{V} \mathrm{m}^{-1}$ ) for all 54 tracts.

1 to $-\lambda^{2} \frac{\partial E_{l}}{\partial l}$ for tract $1^{*}$, both taking place at their beginning points. So a slightly different tract can give rise to the occurrence of other stimulation mechanisms.

For tract 2 , the distance $l$ is measured from coordinate $(-6.11,-10.55,19.57)$ towards $(-13.00,4.31,-29.50)$. A mean $\Delta E_{l}$ of $0.70 \mathrm{~V} \mathrm{~m}^{-1}$ is observed and a maximum of $2.70 \mathrm{~V} \mathrm{~m}^{-1}$ for $l$ equal to $23.9 \mathrm{~mm}$. $\Delta l$ varies from 1.0 to $5.9 \mathrm{~mm}$ and is on average $2.8 \mathrm{~mm}$. For both tracts, $-\lambda E_{l}$ is the dominant mechanism and activation will take place at the beginning of the neural fibres.

These results demonstrate the strong variability of the effective electric field among the tracts and confirm the need to model TMS effects for each case and each patient separately.

\subsection{Neglecting tissue anisotropy}

When comparing the electric field of all cerebral tissues between the isotropic and DTIbased anisotropic head model, differences up to $38.89 \mathrm{~V} \mathrm{~m}^{-1}$ are observed with an average of $0.88 \mathrm{~V} \mathrm{~m}^{-1}$.

Figure 11 depicts the effective electric field along the five selected tracts. At some tract points the field decreases with maximum $6.44 \mathrm{~V} \mathrm{~m}^{-1}$, at others it increases with maximum 




Figure 12. Effect of not well-known conductivity values on the effective electric field (in $\mathrm{V} \mathrm{m}^{-1}$ ). (Upper) Box plots of all 54 tracts of the difference $\Delta E_{l \sigma}$ equal to $E_{l \sigma}-E_{l}$ due to varying $\sigma$. They are arranged with an increasing WM/GM ratio and are labelled with the factors both conductivities $\sigma_{W M} / \sigma_{G M}$ are multiplied with. (Lower) For each case, the corresponding conductivity values (in $\mathrm{S} \mathrm{m}^{-1}$ ) of white and grey matter are indicated with red and blue bars, respectively.

$9.33 \mathrm{~V} \mathrm{~m}^{-1}$, while the spatial field variation remains globally the same. To draw general conclusions, we computed the effective fields along all 54 tracts, resulting in a total of 41664 data points, and generated box plots for both the anisotropic and isotropic case and the difference between both $\left(\Delta E_{\text {liso }}=E_{\text {liso }}-E_{l}\right)$, see figure 11. On each box, the central red mark is the median, the edges of the box are the 25th (Q1) and 75th percentiles (Q3), the whiskers extend to extreme data points not considered outliers. Points are defined as outliers if they are smaller than $\mathrm{Q} 1-1.5(\mathrm{Q} 3-\mathrm{Q} 1)$ or larger than $\mathrm{Q} 3+1.5(\mathrm{Q} 3-\mathrm{Q} 1)$, and they are plotted individually. Neglecting tissue anisotropy causes a moderated median increase of $0.29 \mathrm{~V} \mathrm{~m}^{-1} .25 \%$ of all considered points show a decrease of more than $0.73 \mathrm{~V} \mathrm{~m}^{-1}$ and another $25 \%$ an increase of more than $1.87 \mathrm{~V} \mathrm{~m}^{-1}$. More than $90 \%$ of the data points have a $\Delta E_{\text {liso }}$ between -4.64 and $5.78 \mathrm{~V} \mathrm{~m}^{-1}$. The most extreme changes are -10.89 and $10.67 \mathrm{~V} \mathrm{~m}^{-1}$. In general, a localized effective electric field increase is observed due to isotropic tissues. A correlation coefficient of 0.9473 between both cases is obtained.

\subsection{Not well-known conductivity values}

At low frequencies, where the conductivity values in literature are scarce and have larger than average uncertainties, the 4-Cole-Cole model should be used with caution in the knowledge that it provides a best estimate based on present data (Cole and Cole 1941, Gabriel et al 1996). For the applied fundamental frequency $1 / 0.230=4.348 \mathrm{kHz}$, the conductivity values according to this model are 0.066 and $0.108 \mathrm{~S} \mathrm{~m}^{-1}$ for white and grey matter, respectively.

To investigate the effect of these not well-known values, white matter conductivity was multiplied by $0.8,1.0$ and 1.2 and the same variation was applied for grey matter. For each combination, a box plot was generated of $\Delta E_{l \sigma}$, defined as the effective electric field of the adapted minus standard case for all 54 tracts, and all plots are shown in figure 12. They are arranged according to an increasing WM/GM conductivity ratio from 0.41 to 0.92 . At first glance, overall limited differences can be observed. More than $90 \%$ of the data points have a $\Delta E_{l \sigma}$ between -0.66 and $0.67 \mathrm{~V} \mathrm{~m}^{-1}$ and a high correlation coefficient of 0.9981 between $E_{l}$ and $E_{l \sigma}$ is observed. The major changes take place when multiplying white matter conductivity by 1.2 and keeping grey matter conductivity unaltered, with a maximum effective field 

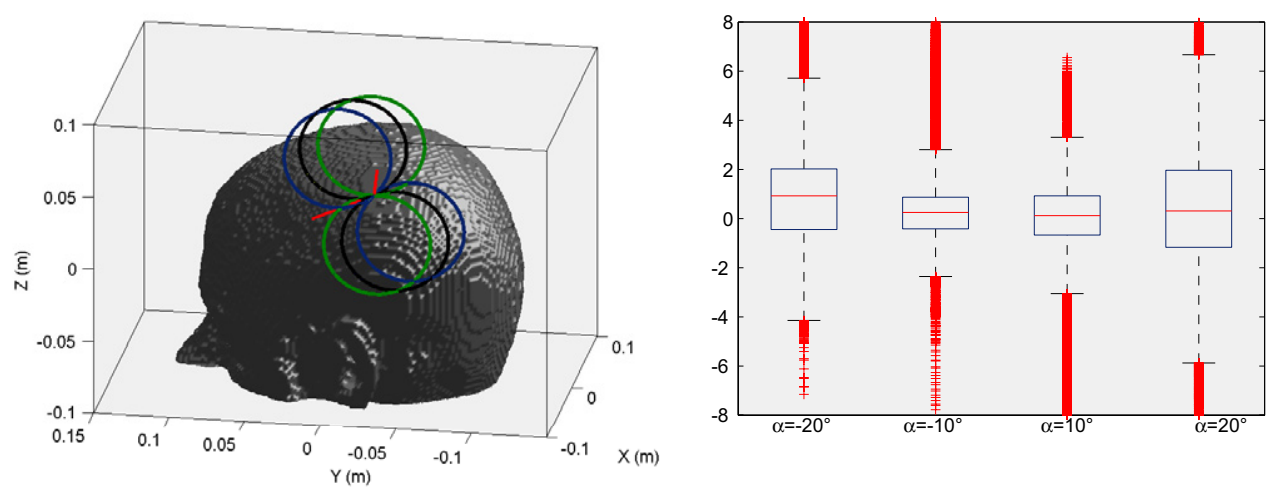

Figure 13. Effect of changing the coil rotation angle $\alpha$. (Left) The red lines illustrate the coil normal and the induced current direction. Rotating the coil through a positive angle $\alpha=20^{\circ}$ about the normal vector is shown in blue and through a negative angle $\alpha=-20^{\circ}$ in green. (Right) Box plots of all 54 tracts of the error $E_{l \alpha}-E_{l}$ due to the different rotations.

increase of $6.35 \mathrm{~V} \mathrm{~m}^{-1}$ and decrease of $6.57 \mathrm{~V} \mathrm{~m}^{-1}$. When looking closer, it is remarkable how the median is always slightly negative (positive) and the 75th percentile is slightly lower (higher) than the 25th percentile in absolute value for reduced (raised) WM/GM conductivity ratios with respect to the original ratio of 0.61 . This means that increasing the ratio resulted in an increased effective electric field and vice versa. However, the impact remains limited and is not proportional for the left M1 hand area stimulation case study.

\subsection{Changes in coil orientation}

In this subsection, we study the influence of changes in the coil orientation to the effective electric field. First, the TMS coil is rotated about the normal vector on the scalp surface through an angle $\alpha$ between -20 to +20 degrees, in steps of 10 degrees. Figure 13 illustrates this coil rotation angle and the field changes $\Delta E_{l \alpha}$ it causes. For $\alpha$ equal to -10 degrees, a moderate median increase of $0.25 \mathrm{~V} \mathrm{~m}^{-1}$ is observed. $25 \%$ of all tract points show a decrease of more than $0.42 \mathrm{~V} \mathrm{~m}^{-1}$ and another $25 \%$ an increase of more than $0.87 \mathrm{~V} \mathrm{~m}^{-1}$. The most extreme changes are -7.78 and $11.75 \mathrm{~V} \mathrm{~m}^{-1}$. For $\alpha$ equal to +10 degrees, a moderate median increase of $0.12 \mathrm{~V} \mathrm{~m}^{-1}$ is observed. $25 \%$ of all points show a decrease of more than $0.67 \mathrm{~V} \mathrm{~m}^{-1}$ and another $25 \%$ an increase of more than $0.92 \mathrm{~V} \mathrm{~m}^{-1}$. The most extreme changes are -12.12 and $6.55 \mathrm{~V} \mathrm{~m}^{-1}$. When the coil is rotated through a larger angle, the differences in effective field obviously increase, leading to larger interquartile ranges and more extreme $\Delta E_{l \alpha}$ up to $20.61 \mathrm{~V} \mathrm{~m}^{-1}$ for $\alpha=-20^{\circ}$ and $-24.08 \mathrm{~V} \mathrm{~m}^{-1}$ for $\alpha=+20^{\circ}$, respectively. More than $90 \%$ of all data points have a $\Delta E_{l \alpha}$ between -2.63 and $3.01 \mathrm{~V} \mathrm{~m}^{-1}$ for rotating the coil 10 degrees and the correlation coefficient amounts 0.9721 . Rotating the coil 20 degrees reduces the latter to 0.8935 .

Secondly, we tilted the TMS coil about the induced current direction through an angle $\beta$, changing from -10 to +10 degrees, in steps of 5 degrees. When increasing the tilt angle even more, the coil crosses the modelled head surface, which is not relevant to simulate. The effect of this tilt angle on the effective electric field $\Delta E_{l \beta}$, defined as $E_{l \beta}$ minus standard $E_{l}$ with $\beta=0^{\circ}$, is presented in box plots in figure 14. A negative angle results in a moderate median 

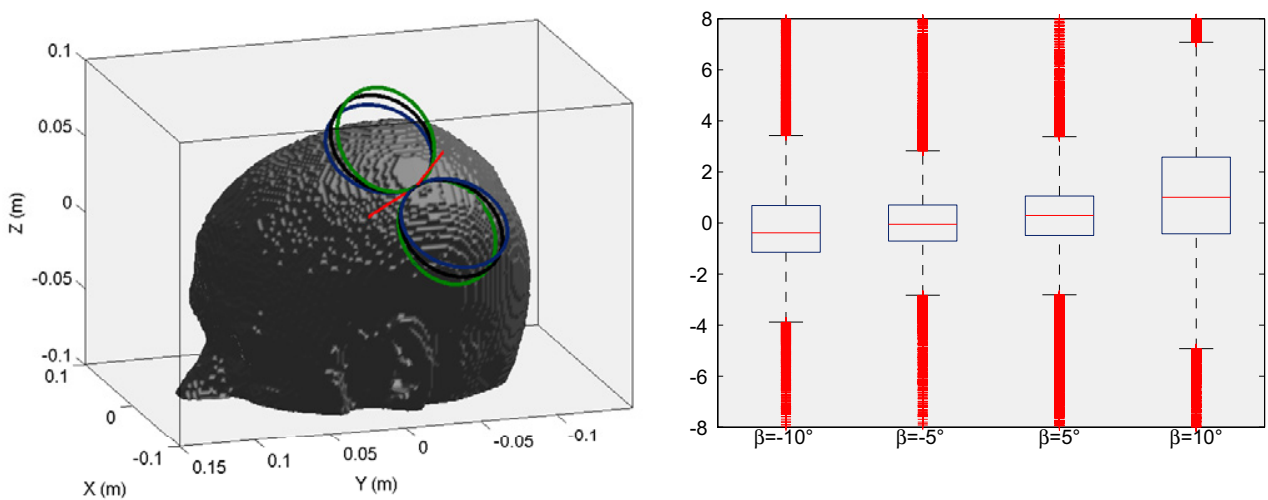

Figure 14. Effect of changing the coil tilt angle $\beta$. (Left) The red lines illustrate the coil normal and the induced current direction. Tilting the coil through a positive angle $\beta=10^{\circ}$ about the induced current direction is shown in blue and through a negative angle $\beta=-10^{\circ}$ in green. (Right) Box plots of all 54 tracts of the error $E_{l \beta}-E_{l}$ due to tilting.

decrease of $0.39 \mathrm{~V} \mathrm{~m}^{-1}\left(\beta=-10^{\circ}\right)$ and $0.05 \mathrm{~V} \mathrm{~m}^{-1}\left(\beta=-5^{\circ}\right)$, while a positive angle results on average in an increase of $0.29 \mathrm{~V} \mathrm{~m}^{-1}\left(\beta=+5^{\circ}\right)$ and $1.00 \mathrm{~V} \mathrm{~m}^{-1}\left(\beta=+10^{\circ}\right)$. Again, larger interquartile ranges and lower correlation coefficients (0.8956 versus 0.9795$)$ are observed for larger tilt angles and more extreme differences up to $14.66 \mathrm{~V} \mathrm{~m}^{-1}$ and $-38.59 \mathrm{~V} \mathrm{~m}^{-1}$. More than $90 \%$ of the data points have a $\Delta E_{l \beta}$ between -4.99 and $5.73 \mathrm{~V} \mathrm{~m}^{-1}$ for tilting the coil 10 degrees. Consequently, tilting causes almost twice greater changes in the effective electric field than coil rotation for this case study.

\section{Discussion and conclusion}

A flexible solver has been developed for calculating the electromagnetic phenomena of TMS. Patient-specific results are preserved, since the head models include realistic geometry, tissue anisotropy and targeted neural fibre bundles based on MRI and tractography. The induced electric field distribution is computed in the whole brain and its component parallel to the neurons' local orientation, the so-called effective electric field, is studied. Most models use 2D or simplified representations of the neural fibres with assumed geometries, whereas we embedded realistic 3D neural trajectories near the region of interest reconstructed by DTIbased tractography.

A large and irregular spatial spread of the effective electric field is observed. Up to $7 \mathrm{~cm}$ distance from the M1 ROI centre, fields of $6 \mathrm{~V} \mathrm{~m}^{-1}$ are induced at some points along the tracts (figure 8). Moreover, strong and localized field variations between the tracts themselves and along them are demonstrated. This is caused by the interplay of factors such as the tract's position and orientation in relation to the TMS coil, the neural trajectory and its course along the white and grey matter interface. The effective electric field term $-\lambda E_{l}$ proved to be the most dominant stimulation mechanism in the 5 main fibre bundles, occurring at neural terminations and bends (figure 9). The directional derivative term $-\lambda^{2} \frac{\partial E_{l}}{\partial l}$ could compete at certain tract points, for example at the beginning points of tract $1 *$ (figure 10), whereas the jump term $-\lambda \frac{\Delta E}{2}$ remained small $(<3 \mathrm{mV})$ for all tracts. 
However, the obtained findings should not be extrapolated without caution. It is not the aim of this paper to draw general conclusions about which tracts or broader regions will be stimulated due to TMS and which stimulation mechanisms will cause the effect. All simulations of this paper were performed for one specific healthy volunteer (female, $25 \mathrm{y}$ ), using her personal structural T1-weighted and diffusion-weighted MRI, and with single pulse stimulation delivered to the left M1 hand area as case study. Nevertheless, we can make conclusions about the sensitivity of the model towards the investigated parameters.

Whereas it has been suggested that tissue anisotropy has only a modest effect on TMS modelling and consequently it can be ignored without major errors (Opitz et al 2011, Laakso et al 2014, Nummenmaa et al 2014), our study indicates that these errors have the same order of magnitude as rotating the stimulation coil with 10 degrees, displayed in the box plots of figures 11, 13 and 14. Therefore, we recommend the incorporation of realistic DTI-based anisotropy. The impact of including not-well known conductivity values, on the other hand, is limited, see figure 12, and hence justifies the use of the less accurate 4-Cole-Cole model for the $\mathrm{kHz}$ range. Moreover, we observed that changing these different parameters affected the effective electric field and corresponding stimulation mechanisms in a non-trivial way. The interplay of their effects give rise to non-linear complex outcomes. It is therefore hard to draw general conclusions on the effects of parameters on the effective electric field. Furthermore, a strong variability can be observed. Consequently, predictions on stimulation results are difficult to make and case- and patient-specific simulations accommodating all parameters and respective effects are essential.

In this work, the diffusion MRI analysis was based on DTI, which is not adequate to characterize complex fibre configurations, such as crossing and kissing fibres (Tournier et al 2011, Jones et al 2013). In these regions, the uncertainty of the dominant diffusion orientation is large, which makes it difficult to reliably reconstruct white matter fibre pathways (Deprez et al 2013, Jeurissen et al 2013). Future research that can integrate such dispersion of pathway propagation during tractography, for instance by resorting to probabilistic approaches (Behrens et al 2007, Jones 2008, Jeurissen et al 2011), is needed to tackle these limitations. This study focussed on 54 selected neural fibre tracts, derived from 3D tractography reconstruction. One of the limitations is the missing interneurons, which are assumed to be indirectly excited by TMS (Di Lazzaro and Ziemann 2013). The effects of incorporating shorter tracts $(<5 \mathrm{~cm})$, applying tractography with lower FA threshold or higher bending angle threshold should be analysed, together with the impact of a higher stimulator output, in order to exceed the stimulation threshold of $52 \mathrm{mV}$ (Silva et al 2008). Computing the spatial and temporal variation of the membrane potentials of the considered neural tracts, based on the stimulation mechanisms discussed in this paper, might be a future research step. Validation of TMS modelling with clinical measurements remains a challenge, but can be done in animal studies.

\section{Acknowledgments}

This work was carried out using the Stevin Supercomputer Infrastructure at Ghent University. N De Geeter gratefully acknowledges the financial support of the Agency for Innovation by Science and Technology in Flanders (IWT) SB/101133. G Crevecoeur acknowledges the Fund of Scientific Research Flanders (FWO). The research of A Leemans is supported by VIDI Grant 639.072.411 from the Netherlands Organisation for Scientific Research (NWO). 


\section{Appendix A.}

A realistic personalised head model is reconstructed based on the patient's MRI. The DTI data set was coregistered to the T1-weighted data, leading to $160 \times 256 \times 256$ voxel matrices with voxelsize $(1,0.9375,0.9375) \mathrm{mm}^{3}$. The coordinate system $(\mathrm{x}, \mathrm{y}, \mathrm{z})$ is defined according to the neurological convention. The $x$-axis is oriented from left $(\mathrm{L})$ to right $(\mathrm{R})$, the $y$-axis from posterior (P) to anterior (A) and the $z$-axis from inferior (I) to superior (S), as can be seen in figure 4 . Since the coil is positioned above the left M1 hand area, we extended the matrices by adding 32 voxels air to the left of the head (neg $\mathrm{x}$ ) and 20 voxels air superiorly (pos z), in order to reduce simulation inaccuracies. On the other hand, we cut off 4 voxels air anteriorly (pos y) and 56 voxels tissue below the nose (neg z), so to prevent unnecessary calculations in less interesting regions. This resulted in $192 \times 252 \times 220$ matrices, or FOV of $192 \times 236.25 \times 206.25 \mathrm{~mm}^{3}$, with the origin of the coordinate system $(0,0,0)$ in the middle, or more specifically at the lower left hand rear corner of voxel $(96,126,110)$. To reduce the computation time and memory of the electromagnetic solver, the voxelsize of the head model was doubled for the simulations, leading to induced electric field distributions with matrix size $96 \times 126 \times 110$.

Whole brain tractography is performed based on the $1 \mathrm{~mm}$ DTI, using ExploreDTI. During this deterministic tracking algorithm, neural trajectories between 5 and $25 \mathrm{~cm}$ are traced starting from each voxel with $1 \mathrm{~mm}$ step size until FA becomes smaller than 0.2 or the direction changes more than 30 degrees. Although there is a user-defined influence, these main tractography parameters are in line with the conventional settings range in current literature (e.g. Lebel et al 2008 and Caeyenberghs and Leemans 2014). Since TMS targets the left M1 hand area in this paper, this determines the central seed ROI, with coordinates $(-0.0210,-0.0216,0.0234)$ in $\mathrm{mm}$. Around this point a $31 \times 31 \times 31$-voxel ROI box is defined ( 15 voxels extra in each direction). Subsequently, the ROI method is applied in ExploreDTI, meaning all 12797 tracts traversing this box are extracted from the whole-brain tractogram. Only those tracts whose first and last points are located less than $10 \mathrm{~mm}$ from another are kept, leading to 285 tracts. The subsequent selection criteria are that both end points should be connected to a GM-WM interface within their $5 \times 5 \times 5$-voxel environment ( 2 voxels extra in each direction), and that no other tissue than white matter should be present along the tract between those two interfaces, see figure 3 for tract 1 and 4 . This resulted in a total of 54 tracts, of which 7 have more than 50 representations. From these 7 main tracts, 5 have differently and 2 similarly oriented trajectories. They are represented as 3D space curves, i.e. $x$-, $y$ - and $z$-coordinates, and coregistered with the previous described reference space (ExploreDTI applies the radiological (AP, RL, IS) coordinate system). The orientation of all considered neural fibre tracts is shown in figure 2 together with their position relative to the seed ROI, of which the centre is indicated with a blue dot.

\section{References}

Barker A, Jalinous R and Freeston I 1985 Noninvasive magnetic stimulation of human motor cortex Lancet 2 1106-7

Basser P J, Pajevic S, Pierpaoli C, Duda J and Aldroubi A 2000 In vivo fiber tractography using DT-MRI data Magn. Reson. Med. 44 625-32

Behrens T E, Berg H J, Jbabdi S, Rushworth M F and Woolrich M W 2007 Probabilistic diffusion tractography with multiple fibre orientations: what can we gain? NeuroImage 34 144-55

Caeyenberghs K and Leemans A 2014 Hemispheric lateralization of topological organization in structural brain networks Hum. Brain Mapp. 35 4944-57 
Cohen L G, Roth B J, Nilsson J, Dang N, Panizza M, Bandinelli S, Friauf W and Hallett M 1990 Effects of coil design on delivery of focal magnetic stimulation. Technical considerations Electroencephalogr. Clin. Neurophysiol. 75 350-7

Cole K S and Cole R H 1941 Dispersion and absorption in dielectrics: alternating current characteristics J. Chem. Phys. $9341-51$

De Geeter N, Crevecoeur G and Dupré L 2011a An efficient 3D eddy-current solver using an independent impedance method for transcranial magnetic stimulation IEEE Trans. Biomed. Eng. 58 310-20

De Geeter N, Crevecoeur G and Dupré L 2011b Eddy-current simulations using an independent impedance method in anisotropic biological tissues IEEE Trans. Magn. 47 3845-8

De Geeter N, Crevecoeur G, Dupré L, Van Hecke W and Leemans A 2012 A DTI-based model for TMS using the independent impedance method with frequency-dependent tissue parameters Phys. Med. Biol. 57 2169-88

De Lucia M, Parker G J, Embleton K, Newton J M and Walsh V 2007 Diffusion-tensor MRI based estimation of the influence of brain tissue anisotropy on the effect of transcranial magnetic stimulation NeuroImage 36 1159-70

Deprez S, Billiet T, Sunaert S and Leemans A 2013 Diffusion tensor MRI of chemotherapy-induced cognitive impairment in non-CNS cancer patients: a review Brain Imag. Behav. 7 409-35

Di Lazzaro V and Ziemann U 2013 The contribution of transcranial magnetic stimulation in the functional evaluation of microcircuits in human motor cortex Front. Neural Circuits 700018

Fregni F, Simon D K, Wu A and Pascual-Leone A 2005 Non-invasive brain stimulation for Parkinson's disease: a systematic review and meta-analysis of the literature J. Neurol. Neurosurg. Psychiatr. 76 1614-23

Friston K J, Ashburner J, Kiebel S J, Nichols T E and Penny W D2007 Statistical Parametric Mapping: the Analysis of Functional Brain Images (London: Academic)

Gabriel S, Lau R W and Gabriel C 1996 The dielectric properties of biological tissues: III. Parametric models for the dielectric spectrum of tissues Phys. Med. Biol. 41 2271-93

Ilmoniemi R J, Ruohonen J and Karhu J 1999 Transcranial magnetic stimulation: a new tool for functional imaging of the brain Crit. Rev. Biomed. Eng. 27 241-84

Irfanoglu M O, Walker L, Sarlls J, Marenco S and Pierpaoli C 2012 Effects of image distortions originating from susceptibility variations and concomitant fields on diffusion MRI tractography results NeuroImage $\mathbf{6 1} 275-88$

Janssen A M, Rampersad S M, Lucka F, Lanfer B, Lew S, Aydin U, Wolters C H, Stegeman D F and Oostendorp T F 2013 The influence of sulcus width on simulated electric fields induced by transcranial magnetic stimulation Phys. Med. Biol. 58 4881-96

Jeurissen B, Leemans A, Jones D K, Tournier J D and Sijbers J 2011 Probabilistic fiber tracking using the residual bootstrap with constrained spherical deconvolution Hum. Brain Mapp. 32 461-79

Jeurissen B, Leemans A, Tournier J D, Jones D K and Sijbers J 2013 Investigating the prevalence of complex fiber configurations in white matter tissue with diffusion magnetic resonance imaging Hum. Brain Mapp. 34 2747-66

Jones D K 2008 Tractography gone wild: probabilistic fibre tracking using the wild bootstrap with diffusion tensor MRI IEEE Trans. Med. Imaging 27 1268-74

Jones D K, Knsche TR and Turner R 2013 White matter integrity, fiber count, and other fallacies: the do's and don'ts of diffusion MRI NeuroImage 73 239-54

Jones D K and Leemans A 2011 Diffusion tensor imaging Methods Mol. Biol. 711 127-44

Laakso I, Hirata A and Ugawa Y 2014 Effects of coil orientation on the electric field induced by TMS over the hand motor area Phys. Med. Biol. 59 203-18

Lebel C, Walker L, Leemans A, Phillips L and Beaulieu C 2008 Microstructural maturation of the human brain from childhood to adulthood NeuroImage 40 1044-55

Leemans A, Jeurissen B, Sijbers J and Jones D K 2009 ExploreDTI: a graphical toolbox for processing, analyzing, and visualizing diffusion MR data 17th Annual Meeting of Int. Society for Magnetic Resonance in Medicine (Hawaii, USA, 18-24 April 2009) p 3537

Leemans A and Jones D K 2009 The B-matrix must be rotated when correcting for subject motion in DTI data Magn. Reson. Med. 61 1336-49

Loo C K and Mitchell P B 2005 A review of the efficacy of transcranial magnetic stimulation (TMS) treatment for depression, and current and future strategies to optimize efficacy J. Affect. Disorders $3255-67$

Lu M, Ueno S, Thorlin T and Persson M 2008 Calculating the activating function in the human brain by transcranial magnetic stimulation IEEE Trans. Magn. 44 1438-41 
Miranda P C, Correia L, Salvador R and Basser P J 2007 Tissue heterogeneity as a mechanism for localized neural stimulation by applied electric fields Phys. Med. Biol. 52 5603-17

Nummenmaa A, McNab J A, Savadjiev P, Okada Y, Hmlinen MS, Wang R, Wald L L, Pascual-Leone A, Wedeen V J and Raij T 2014 Targeting of white matter tracts with transcranial magnetic stimulation Brain Stimulation 7 80-4

Opitz A, Windhoff M, Heidemann R M, Turner R and Thielscher A 2011 How the brain tissue shapes the electric field induced by transcranial magnetic stimulation NeuroImage 58 849-59

Padberg F and George M S 2009 Repetitive transcranial magnetic stimulation of the prefrontal cortex in depression Exp. Neurol. 219 2-13

Pashut T, Wolfus S, Friedman A, Lavidor M, Bar-Gad I, Yeshurun Y and Korngreen A 2011 Mechanisms of magnetic stimulation of central nervous system neurons PLoS Comput. Biol. 7 e1002022

Rossi S, Hallett M, Rossini P M and Pascual-Leone A 2009 Safety, ethical considerations, and application guidelines for the use of transcranial magnetic stimulation in clinical practice and research Clin. Neurophysiol. 120 2008-39

Roth B J and Basser P J 1990 A model of the stimulation of a nerve fiber by electromagnetic induction IEEE Trans. Biomed. Eng. 37 588-97

Sack A T and Linden D E 2003 Combining transcranial magnetic stimulation and functional imaging in cognitive brain research: possibilities and limitations Brain Res. Rev. 43 41-56

Salinas F S, Lancaster J L and Fox P T 2007 Detailed 3D models of the induced electric field of transcranial magnetic stimulation coils Phys. Med. Biol. 52 2879-92

Salvador R, Silva S, Basser P J and Miranda P C 2011 Determining which mechanisms lead to activation in the motor cortex: a modeling study of transcranial magnetic stimulation using realistic stimulus waveforms and sulcal geometry Clin. Neurophysiol. 122 748-58

Sekino M and Ueno S 2004 FEM-based determination of optimum current distribution in transcranial magnetic stimulation as an alternative to electroconvulsive therapy IEEE Trans. Magn. 40 2167-9

Shahid S S, Bikson M, Salman H, Wen P and Ahfock T 2014 The value and cost of complexity in predictive modelling: role of tissue anisotropic conductivity and fibre tracts in neuromodulation J. Neural Eng. 11036002

Silva S, Basser P J and Miranda P C 2008 Elucidating the mechanisms and loci of neuronal excitation by transcranial magnetic stimulation using a finite element model of a cortical sulcus Clin. Neurophysiol. $1192405-13$

Smith J A, Mennemeier M, Bartel T, Chelette K C, Kimbrell T, Triggs W and Dornhoffer J L 2007 Repetitive transcranial magnetic stimulation for tinnitus: a pilot study Laryngoscope 117 529-34

Tax C M, Otte W M, Viergever M A, Dijkhuizen R M and Leemans A 2014 REKINDLE: Robust extraction of kurtosis INDices with linear estimation Magn. Reson. Med. at press (http://dx.doi. $\operatorname{org} / 10.1002 / \mathrm{mrm} .25165)$

Thielscher A and Kammer T 2004 Electric field properties of two commercial figure 9 coils in TMS: calculation of focality and efficiency Clin. Neurophysiol. 115 1697-708

Tournier J D, Mori S and Leemans A 2011 Diffusion tensor imaging and beyond Magn. Reson. Med. $651532-56$

Tuch D S, Wedeen V J, Dale A M, George J S and Belliveau J W 2001 Conductivity tensor mapping of the human brain using diffusion tensor MRI Proc. Natl Acad. Sci. USA 98 11697-701

Veraart J, Sijbers J, Sunaert S, Leemans A and Jeurissen B 2013 Weighted linear least squares estimation of diffusion MRI parameters: strengths, limitations, and pitfalls NeuroImage 81 335-46

Wagner T A, Fregni F, Eden U, Ramos-Estebanez C, Grodzinsky A J, Zahn M and Pascual-Leone A 2006 Transcranial magnetic stimulation and stroke: a computer-based human model study NeuroImage 30 857-70

Wagner T A, Zahn M, Grodzinsky A J and Pascual-Leone A 2004 Three-dimensional head model simulation of transcranial magnetic stimulation IEEE Trans. Biomed. Eng. 51 1586-94

Wang H, Liu F, Xia L and Crozier S 2008 An efficient impedance method for induced field evaluation based on a stabilized Bi-conjugate gradient algorithm Phys. Med. Biol. 53 6363-75 